\title{
Dimensional Properties of Polypeptides in the Helix-Coil Transition Region I. Molecular Dimension
}

\author{
Jong Ryul KIM and Taikyue REE \\ Department of Chemistry, Korea Advanced Institute of Science and Technology, \\ P.O. Box 150, Cheongryangni, Seoul 131, Korea
}

(Received February 3, 1984)

\begin{abstract}
An expression was derived to correlate the unperturbed mean-square radius of gyration of an intermediate polypeptide chain $\left\langle s^{2}\right\rangle_{\mathrm{ol}}$ with helical content $f_{\mathrm{H}}$ (see eq 10). An unperturbed state corresponding to the $\alpha$-helix was obtained by converting the helix to a tortuous random-coil with a bond-rotation angle of $+100^{\circ}$ or $-100^{\circ}$ (in cis-convention) per residue, or per "virtual bond," and a bond angle satisfying the experimental value of 8.8 for the characteristic ratio of the random-coil of PBLG. The "quasi" linear expansion factor $\alpha_{h}$ of a helical sequence in an intermediate chain was evaluated (see eq 18) using the Zimm-Bragg-Nagai theory in collaboration with our expression for $\left\langle s^{2}\right\rangle_{0}$. The intermediate chain may be a kind of swollen randomcoil owing to the existence of helical sequences in the chain, and thus, the linear expansion factor $\alpha_{\mathrm{sI}}$ of the entire intermediate chain was approximately formulated as $\alpha_{\mathrm{SI}}=\alpha_{\mathrm{sC}} \alpha_{\mathrm{h}}$ where $\alpha_{\mathrm{sC}}$ is the linear expansion factor for the radius of gyration of a random-coil. With $\alpha_{\mathrm{sI}}$ and $\left\langle s^{2}\right\rangle_{\mathrm{ol}}$, the mean-square radius of gyration $\left\langle s^{2}\right\rangle_{1}$ of polypeptides in the transition region was calculated (eq 12) and compared with that obtained experiment (see Figure 4). Our theoretical results were found to agree well with experiment.
\end{abstract}

KEY WORDS Solution Properties / Zimm-Bragg-Nagai Theory / HelixCoil Transition / Synthetic Polypeptide / Helical Content / Molecular Dimension / Linear Expansion Factor /

The helix-coil transition of polypeptides ${ }^{1}$ is much interest and importance. Thus, with typical polypeptides as samples, a great many investigations $^{2}$ have been performed to find out how the dimensional properties of polypeptides in dilute solution are affected when a helix-coil transition occurs under various environmental conditions. During the last two decades, there have been several attempts ${ }^{3-8}$ to treat this transition by statistical mechanical methods. A partition function of a polypeptide molecule has been constructed by matrix methods. Explicit formulae have been given ${ }^{8,9}$ for the helical content $f_{\mathrm{H}}$ and the average number of helical sequences $g_{\mathrm{H}}$ as well as their distribution in a polypeptide chain. The Zimm and Bragg theory ${ }^{6}$ has shown good agreement with experiment for the change in optical rotation in the transition region.

The general objective in studying the helixcoil transition is to explain the dependence of dilute solution properties of polypeptides on the helical content $f_{\mathrm{H}}$ and the chain length $n$. The representative conformation-dependent properties are the average molecular dimensions (the mean-square radius of gyration $\left\langle s^{2}\right\rangle$ and the mean-square end-to-end distance $\left\langle r^{2}\right\rangle$ ), the hydrodynamic properties (the intrinsic viscosity $[\eta]$ and the translational friction coefficient) and the mean-square dipole moment $\left\langle\mu^{2}\right\rangle$. Nagai ${ }^{9,10}$ and Miller and Flory ${ }^{11}$ studied the molecular dimensions in the transition region by applying the Zimm-Bragg theory. ${ }^{6}$ But their theoretical results did not provide a complete explanation of the experimental results. ${ }^{12}$ Neves and Scott III $^{13}$ con- 


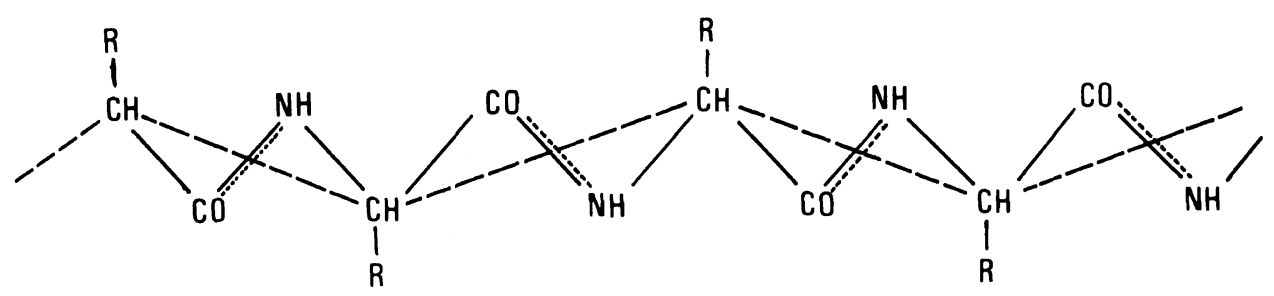

Figure 1. The polypeptide chain. Virtual bonds are shown by dashed lines connecting consecutive $\alpha$ carbon atoms. The partial double-bond character of the amide bond is also evident.

cluded the excluded volume effect to be important for predicting the dimensional properties of polypeptides in the helix-coil transition region.

Thus, as an extension of Nagai's theory, ${ }^{9}$ another model for calculating the unperturbed dimension $\left\langle s^{2}\right\rangle_{0}$ and linear expansion factor for the radius of gyration $\alpha_{s}$ at various stages of the helix-coil transition is proposed in this paper: for $\left\langle s^{2}\right\rangle_{0}$, the internal rotation of polypeptides is taken into account, and $\alpha_{\mathrm{s}}$ is obtained by application of the Zimm-BraggNagai theory. ${ }^{6,9}$ This kind of study, calculating $\alpha_{\mathrm{s}}$ of polypeptides in the helix-coil transition region, has never appeared in the literature. Our results for $\left\langle s^{2}\right\rangle$ were compared with experimental data. The detailed theory, results and discussion are reported in the text.

\section{THEORY}

\section{Unperturbed Dimension}

Brant and Flory ${ }^{14,15}$ treated the polypeptide chain as a sequence of virtual bonds of length $l=3.8 \AA$, a fixed distance between the $\alpha$-carbons of the trans-peptide repeating unit (i.e., $\alpha$ - amino acid residues $-\mathrm{CHR}-\mathrm{CO}-\mathrm{NH}-$ ) in the chain. Virtual bonds are shown in Figure 1 by dashed lines. A peptide unit is in a planar trans-conformation permitting a spatial configuration of the polypeptide chain to be expressed in terms of the virtual bonds, all the bond lengths and bond angles in the repeating unit being essentially fixed. ${ }^{15}$

By the foregoing treatment, the polypeptide chain was simplified by a simple chain comprising identical skeletal atoms joined by identical virtual bonds. (Hereafter the "virtual bond" is represented simply by the "bond.") If the bond-rotational potential is independent of its neighbors, the characteristic ratio $C_{n}$ can be expressed $^{16 a}$ as follows:

$$
\begin{aligned}
C_{n} & \equiv\left\langle r^{2}\right\rangle_{\mathrm{o}} / n l^{2} \\
& =\left[(\boldsymbol{E}+\langle\boldsymbol{T}\rangle)(\boldsymbol{E}-\langle\boldsymbol{T}\rangle)^{-1}\right]_{11}+O\left(n^{-1}\right)
\end{aligned}
$$

where $\left\langle r^{2}\right\rangle_{\mathrm{o}}$ is the unperturbed mean-square end-to-end distance, $n$, the chain length, $\boldsymbol{E}$, the $3 \times 3$ identity matrix, $\langle\boldsymbol{T}\rangle$, the average transformation matrix, and the subscript 11 denotes the 1,1 element of the matrix product. $\langle\boldsymbol{T}\rangle$ is given by the following equation: ${ }^{16 a}$

$$
\langle\boldsymbol{T}\rangle=\left[\begin{array}{ccc}
\cos \theta & \sin \theta & 0 \\
\sin \theta\langle\cos \phi\rangle & -\cos \theta\langle\cos \phi\rangle & \langle\sin \phi\rangle \\
\sin \theta\langle\sin \phi\rangle & -\cos \theta\langle\sin \phi\rangle & -\langle\cos \phi\rangle
\end{array}\right]
$$

where $\theta$ and $\phi$ are the bond angle and bondrotation angle (in trans-convention), respectively.
The randomly coiled state of the polypeptide is set as a freely rotating chain in this paper. The real random-coil of polypeptides 
however is not exactly identical to the freely rotating chain, since there is some rotational hindrance, etc. Thus, our $\theta$ is not a real value, ${ }^{16 \mathrm{~b}}$ but a hypothetical bond angle satisfying the experimental value ${ }^{14}$ of 8.8 for $\left\langle r^{2}\right\rangle_{\mathrm{oC}} \mid$ $n l^{2}$ (see eq 6 below). If the real value of $\theta$ is used, the calculated value of $\left\langle r^{2}\right\rangle_{\mathrm{oC}} \mid$ $n l^{2}\left(=1.93^{16 \mathrm{~b}}\right)$ is much less than the experimental value.

1. $\alpha$-Helical Residue. The polypeptide helix is an $\alpha$-helix, ${ }^{17}$ i.e., 3.6 residues per turn of the helix. Every bond rotates with the same magnitude and the same sense in the helical conformation so that a residue has the same spatial orientation with respect to the 3.6 th preceding residue in the $\alpha$-helix. If the bondrotation angle per turn, or per 3.6 virtual bonds, is assumed to be $360^{\circ}$ (cis-convention), the mean bond-rotation angle should be $100^{\circ}$ $\left(=360^{\circ} / 3.6\right.$, cis-convention $)$ or $80^{\circ}(=180$ $100^{\circ}$, trans-convention). Ptitsyn and Sharanov $^{18}$ successfully obtained the unperturbed dimension of the helical conformation of polyisobutylene by converting ${ }^{18,19}$ the helix to a tortuous random-coil by reversing the signs of bond rotation along the entire chain without altering the magnitudes of the rotations, ${ }^{19}$ where the signs of bond rotation are independent of the neighboring bonds. Hence, in order to consider the unperturbed state of the regular $\alpha$-helix chain, the same method is used in this paper. The bond-rotation angle of the tortuous random-coil corresponding to the unperturbed state of $\alpha$-helix is $\pm 100^{\circ}$ (cis-convention) or $\pm 80^{\circ}$ (transconvention) for every bond, and $\langle\cos \phi\rangle=$ $\cos 80^{\circ},\left\langle\sin \left( \pm 80^{\circ}\right)\right\rangle=0$. Hence, from eq 2 , we obtain,

$$
\langle\boldsymbol{T}\rangle_{\mathbf{H}}=\left[\begin{array}{ccc}
\cos \theta & \sin \theta & 0 \\
\sin \theta \cos 80^{\circ} & -\cos \theta \cos 80^{\circ} & 0 \\
0 & 0 & -\cos 80^{\circ}
\end{array}\right]
$$

The $\langle\boldsymbol{T}\rangle_{\mathrm{H}}$ is the transformation matrix for the $\alpha$-helical residue which represents the unperturbed state of $\alpha$-helix, i.e., the tortuous random-coil mentioned above. Substitution of eq 3 into eq 1 yields

$$
\frac{\left\langle r^{2}\right\rangle_{\mathrm{oH}}}{n l^{2}}=\left(\frac{1+\cos \theta}{1-\cos \theta}\right)\left(\frac{1+\cos 80^{\circ}}{1-\cos 80^{\circ}}\right)+O\left(n^{-1}\right)
$$

where $\left\langle r^{2}\right\rangle_{\mathrm{oH}}$ is the mean-square end-to-end distance of the tortuous random-coil ${ }^{18,19}$ corresponding to the unperturbed state of $\alpha$-helix.

2. Randomly Coiled Residue. The randomcoil of polypeptides is set to be a freely rotating chain, i.e., $\langle\cos \phi\rangle=\langle\sin \phi\rangle=0$, which is closer to a real random-coil than the freely jointed chain, the former being more often used for describing the random-coil. ${ }^{20 a}$ Thus eq 2 reduces to ${ }^{16 a}$

$$
\langle\boldsymbol{T}\rangle_{\mathrm{C}}=\left[\begin{array}{ccc}
\cos \theta & \sin \theta & 0 \\
0 & 0 & 0 \\
0 & 0 & 0
\end{array}\right]
$$

where $\langle\boldsymbol{T}\rangle_{\mathrm{C}}$ is the transformation matrix for the randomly coiled residue. Substitution of eq 5 into eq 1 leads directly to the following well-known equation: ${ }^{16 c}$

$$
\frac{\left\langle r^{2}\right\rangle_{\mathrm{oC}}}{n l^{2}}=\left(\frac{1+\cos \theta}{1-\cos \theta}\right)+O\left(n^{-1}\right)
$$

Here $\left\langle r^{2}\right\rangle_{\mathrm{oC}}$ has the same meaning as the $\left\langle r^{2}\right\rangle_{\mathrm{o}}$ in eq 1 , but in order to emphasize a randomly coiled state, the subscript $\mathrm{C}$ is attached. Since $\theta$ is the bond angle as mentioned previously, it should have a fixed value in the helix-coil transition region.

3. Intermediates in the Transition Region. An intermediate chain is composed of two 
kinds of residues, $\alpha$-helical and randomly coiled residues. The unperturbed dimension of the intermediate chain may be obtained in the absence of interaction between the two kinds of residues. Hence, the average transformation matrix $\langle\bar{T}\rangle$ for an intermediate may be written $\mathrm{as}^{20 \mathrm{~b}}$

$$
\langle\overline{\boldsymbol{T}}\rangle=\left(1-f_{\mathrm{H}}\right)\langle\boldsymbol{T}\rangle_{\mathrm{C}}+f_{\mathrm{H}}\langle\boldsymbol{T}\rangle_{\mathrm{H}}
$$

where the helical content $f_{\mathrm{H}}$ represents the fraction of the $\alpha$-helical residue in an intermediate chain. Substitution of eq 3 and 5 into eq 7 yields

$$
\langle\overline{\boldsymbol{T}}\rangle=\left[\begin{array}{ccc}
\cos \theta & \sin \theta & 0 \\
\sin \theta\left(\cos 80^{\circ}\right) f_{\mathrm{H}} & -\cos \theta\left(\cos 80^{\circ}\right) f_{\mathrm{H}} & 0 \\
0 & 0 & -\left(\cos 80^{\circ}\right) f_{\mathrm{H}}
\end{array}\right]
$$

From eq 1 and 8 , we find

$$
\frac{\left\langle r^{2}\right\rangle_{\mathrm{oI}}}{n l^{2}}=\left(\frac{1+\cos \theta}{1-\cos \theta}\right)\left(\frac{1+f_{\mathrm{H}} \cos 80^{\circ}}{1-f_{\mathrm{H}} \cos 80^{\circ}}\right)+O\left(n^{-1}\right)
$$

This is the characteristic ratio of an intermediate chain in the unperturbed state, and used frequently in the following for representing the unperturbed dimension of an intermediate chain (see eq 10, 12, and 20). The second terms on the right sides of eq 4,6 , and 9 are negligible for most experimental conditions of polypeptides $\left(n>10^{2}\right)$. Hence, the relation, $\left\langle r^{2}\right\rangle_{0}=6\left\langle s^{2}\right\rangle_{\mathrm{o}}$, is obtained ${ }^{20 c}$ where $\left\langle s^{2}\right\rangle_{0}$ is the unperturbed mean-square radius of gyration. Division of eq 9 by eq 6 gives the ratio of the unperturbed dimension of an intermediate chain to that of the random-coil, i.e.,

$$
\frac{\left\langle r^{2}\right\rangle_{\mathrm{oI}}}{\left\langle r^{2}\right\rangle_{\mathrm{oC}}}=\frac{\left\langle s^{2}\right\rangle_{\mathrm{oI}}}{\left\langle s^{2}\right\rangle_{\mathrm{oC}}}=\frac{1+f_{\mathrm{H}} \cos 80^{\circ}}{1-f_{\mathrm{H}} \cos 80^{\circ}}
$$

where $\left\langle r^{2}\right\rangle_{\mathrm{oI}}$ and $\left\langle s^{2}\right\rangle_{\mathrm{ol}}$ are the quantities $\left\langle r^{2}\right\rangle_{0}$ and $\left\langle s^{2}\right\rangle_{0}$ of the intermediate chain, respectively, and $\left\langle s^{2}\right\rangle_{\mathrm{OC}}$ is the quantity $\left\langle s^{2}\right\rangle_{\mathrm{o}}$ in the randomly coiled state.

\section{Expansion Factor for a Perturbed Intermediate Chain}

The actual value of the mean-square radius of gyration $\left\langle s^{2}\right\rangle$ is defined as

$$
\left\langle s^{2}\right\rangle \equiv \alpha_{\mathrm{s}}^{2}\left\langle s^{2}\right\rangle_{\mathrm{o}}
$$

where $\alpha_{\mathrm{s}}$ is the linear expansion factor for the radius of gyration which represents the linear perturbation due to the so-called excluded volume effect. We may rewrite the meansquare radius of gyration as

$$
\begin{aligned}
\frac{\left\langle s^{2}\right\rangle_{\mathrm{I}}}{\left\langle s^{2}\right\rangle_{\mathrm{C}}} & =\left(\frac{\alpha_{\mathrm{SI}}}{\alpha_{\mathrm{SC}}}\right)^{2} \frac{\left\langle s^{2}\right\rangle_{\mathrm{oI}}}{\left\langle s^{2}\right\rangle_{\mathrm{oC}}} \\
& =\left(\frac{\alpha_{\mathrm{sI}}}{\alpha_{\mathrm{sC}}}\right)^{2}\left(\frac{1+f_{\mathrm{H}} \cos 80^{\circ}}{1-f_{\mathrm{H}} \cos 80^{\circ}}\right)
\end{aligned}
$$

where eq 10 and 11 are used. In eq 12, $\left\langle s^{2}\right\rangle_{1}$ and $\left\langle s^{2}\right\rangle_{C}$ are $\left\langle s^{2}\right\rangle$ of the intermediate chain and the random-coil, respectively, and $\alpha_{\mathrm{sI}}$ and $\alpha_{\mathrm{sC}}$ are $\alpha_{\mathrm{s}}$ of the intermediate chain and random-coil, respectively.

On using the equation for $\left\langle s^{2}\right\rangle$ of a rigid rod molecule, ${ }^{21}$ the spatial average dimension of an $\alpha$-helix rod of $n$ residues $\left\langle s^{2}\right\rangle_{\mathrm{H}}$ (in $\AA^{2}$ ) is expressed by

$$
\left\langle s^{2}\right\rangle_{\mathrm{H}}=(1 / 12)(1.5 n)^{2}
$$

Here, $1.5 \AA$ is the pitch per monomer residue of the $\alpha$-helix.

By the Zimm-Bragg-Nagai theory, ${ }^{6,9}$ the average number of helical sequences $g_{\mathrm{H}}$ in an intermediate polypeptide chain of $n$ residues in the helix-coil transition region is represented ${ }^{22}$ by

$$
g_{\mathrm{H}}=\left(n \sigma^{1 / 2}\right) f^{1 / 2}(1-f)^{1 / 2}+f(2 f-1)
$$

where $\sigma$ is referred to as the cooperativity parameter $^{6}$ for the formation of helical se- 
quences, or as the helix-initiation parameter, and depends on the polypeptide and solvent. ${ }^{2}$ The quantity $f$ is the parameter related to the degree of transition (random-coil $\rightarrow \alpha$-helix), and is reduced to $f_{\mathrm{H}}$ at $n=\infty$ (see below). The average number of residues per helical sequence $n_{\mathrm{h}}$ is expressed by

$$
n_{\mathrm{h}}=n f_{\mathrm{H}} / g_{\mathrm{H}}
$$

and $f_{\mathbf{H}}$ is given ${ }^{23}$ by

$$
f_{\mathrm{H}}=f-2 f^{3 / 2}(1-f)^{1 / 2} /\left(n \sigma^{1 / 2}\right)
$$

as derived from the Zimm-Bragg-Nagai theory. ${ }^{6,9}$

Since the helical sequence is a kind of $\alpha$-helix rod of $n_{\mathrm{h}}$ residues (see eq 15), its $\left\langle s^{2}\right\rangle$ and $\left\langle s^{2}\right\rangle_{\mathrm{o}}$ could be evaluated respectively from eq 13 and 4, where the chain length $n$ should be replaced by $n_{\mathrm{h}}$, and the relation ${ }^{20 \mathrm{c}}\left\langle r^{2}\right\rangle_{\mathrm{o}}=$ $6\left\langle s^{2}\right\rangle_{\text {o }}$ for $\left.n\right\rangle 100$ should be used. The "quasi", expansion factor of the helical sequence $\alpha_{\mathrm{h}}$ in an intermediate chain is then obtained from eq 11 as

$$
\alpha_{\mathrm{h}}^{2}=\frac{\alpha_{\mathrm{h}}^{2}=\left\langle s^{2}\right\rangle_{\mathrm{h}} /\left\langle s^{2}\right\rangle_{\mathrm{oh}}}{\frac{1}{6} n_{\mathrm{h}} l^{2}\left(\frac{1+\cos \theta}{1-\cos \theta}\right)\left(\frac{1+\cos 80^{\circ}}{1-\cos 80^{\circ}}\right)}
$$

where $\left\langle s^{2}\right\rangle_{\mathrm{h}}$ and $\left\langle s^{2}\right\rangle_{\text {oh }}$ are the quantities of $\left\langle s^{2}\right\rangle$ and $\left\langle s^{2}\right\rangle_{\text {o }}$ of the helical sequence in an intermediate chain, respectively. Here, we note that in the derivation of eq $17 \mathrm{~b}$, eq 4 was utilized for the unperturbed dimension of the helical sequence.

The randomly coiled sequences in an intermediate chain act as flexible joints, and hence the intermediate chain should be similar to the random-coil, but, at a considerably large $f_{\mathrm{H}}$, behave as a rod-like molecule rather than a random-coil. In this paper, the intermediate chain whose molecular dimensional feature does not differ very much from random-coils is considered, but it should persist in most of the transition region. Thus, the intermediate chain of $f_{\mathrm{H}}$ not close to unity is assumed to be an expanded random-coil as the random-coil (of $\alpha_{\mathrm{s}}=\alpha_{\mathrm{sC}}$ ) swollen by the amount of $\alpha_{h}$ by the helical sequences (of $\alpha_{s}=$ $\alpha_{h}$ ) formed in the chain (see Figure 2). Since the average helical sequences should occur at any position of the intermediate chain with equal probability, the expansion $\alpha_{\mathrm{h}}$ might be a uniform expansion. The linear expansion factor of the intermediate chain $\alpha_{s I}$ is approximately formulated as

$$
\alpha_{\mathrm{sI}}=\alpha_{\mathrm{sC}} \alpha_{\mathrm{h}}
$$

At a considerably large $f_{\mathrm{H}}$ (i.e., rod-like polypeptides), eq 18 may overestimate the expansion factor $\alpha_{\mathrm{sI}}$ since the factor $\alpha_{\mathrm{sC}}$ in eq 18 should reduce to unity in this case. But eq 18 may be useful for describing the changing trend of the expansion factor in the transition region for $f_{\mathrm{H}}$ not close to unity. Substitution of eq 18 into 12 yields

$$
\frac{\left\langle s^{2}\right\rangle_{\mathrm{I}}}{\left\langle s^{2}\right\rangle_{\mathrm{C}}}=\alpha_{\mathrm{h}}{ }^{2}\left(\frac{1+f_{\mathrm{H}} \cos 80^{\circ}}{1-f_{\mathrm{H}} \cos 80^{\circ}}\right)
$$

The factor, $(1+\cos \theta) /(1-\cos \theta)$, in the denominator of eq $17 \mathrm{~b}$ corresponds to the characteristic ratio $\left\langle r^{2}\right\rangle_{\mathrm{oc}} / n l^{2}$ of polypeptides in the randomly coiled state as mentioned previously (see eq 6). Brant and Flory ${ }^{14}$ determined its value as equal to 8.8 for a high molecular weight poly $(\gamma$-benzyl L-glutamate) (PBLG). Therefore, eq $17 \mathrm{~b}$ reduces to

$$
\alpha_{\mathrm{h}}^{2}=0.0062 n_{\mathrm{h}}
$$

where the value ${ }^{14,15}$ of $l=3.8 \AA$ is used. The expansion factor in eq $20 \mathrm{a}$ should be either larger or smaller than unity, depending on $n_{\mathrm{h}}$. On the basis of the experimental fact (ref 2) that the molecular dimensional properties of polypeptides do not decrease with increasing $f_{\mathrm{H}}$ in the helix-coil transition region for the system of a sufficiently high molecular weight polypeptide and $\sigma \simeq 10^{-4}$, we assumed that the expansion factor of the intermediate chain $\alpha_{\mathrm{sI}}$ (see eq 18) does not decrease with increasing $f_{\mathrm{H}}$ for such a polypeptide-solvent system. Thus, along with eq 18 , all $\alpha_{\mathrm{h}}$ 's less than unity (i.e., $n_{\mathrm{h}}<160$, see eq 20a) are assigned to unity, i.e., 


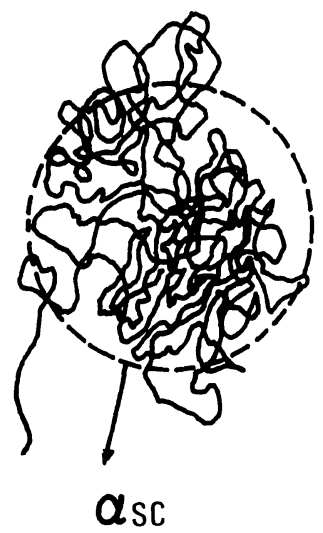

(a)

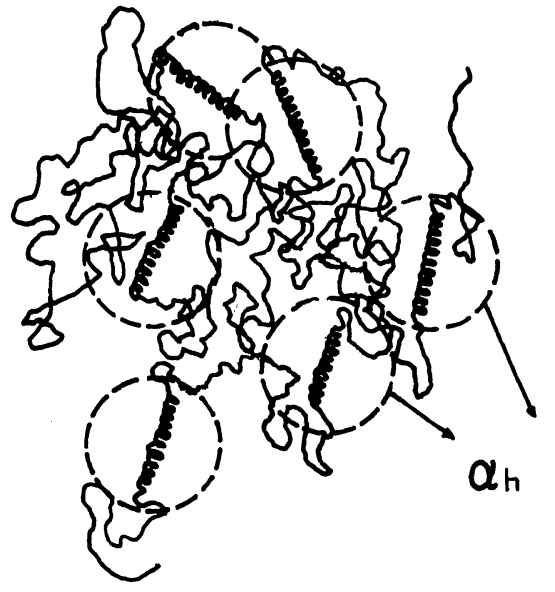

(b)

Figure 2. Schematic representation of a polypeptide chain: (a) randomly coiled form; (b) intermediate chain. $\alpha_{\mathrm{sC}}$ is the expansion factor for the radius of gyration of the random-coil and $\alpha_{\mathrm{h}}$, the expansion factor for the radius of gyration of the average helical sequence (in which $n_{\mathrm{h}} \geq 160$ ) in an intermediate chain.

$$
\alpha_{\mathrm{h}}^{2}=1.0, \quad \text { if } \quad n_{\mathrm{h}}<160
$$

The reason for this can be explained as follows: at $n_{\mathrm{h}}<160$, the helical content $f_{\mathrm{H}}$ is relatively small for a system of sufficiently large $n$ and $\sigma \simeq 10^{-4}$ (see eq 14 to 16), where the randomly coiled sequences should be much more extended than the helical sequences and thus the expansion factor $\alpha_{h}$ of the helical sequences of $n_{\mathrm{h}}<160$ might be screened by expansion due to the randomly coiled sequences in the chain.

\section{RESULTS AND DISCUSSION}

The quantities, $f_{\mathrm{H}}, \quad\left(\left\langle s^{2}\right\rangle_{\mathrm{I}} /\left\langle s^{2}\right\rangle_{\mathrm{C}}\right)^{1 / 2}$, and $\alpha_{\mathrm{sI}} / \alpha_{\mathrm{sC}}\left(=\alpha_{\mathrm{h}}\right)$ for $n=950$ at various given $f$ were calculated using eq 16,19 , and 20 , respectively, with the aid of eq 14 and 15 , and with $\sigma=$ $2.0 \times 10^{-4}$. ${ }^{6}$ The results are shown in Table I. The conformation-dependent property $\left\langle s^{2}\right\rangle_{1}$ in Table I increases slowly over the range from $f_{\mathrm{H}}=0$ to about 0.85 , but increases sharply for $f_{\mathrm{H}}>0.85$. This is due to the trend of the linear
Table I. Dependence of $f_{\mathrm{H}},\left(\left\langle s^{2}\right\rangle_{\mathrm{I}} /\left\langle s^{2}\right\rangle_{\mathrm{C}}\right)^{1 / 2}$, and $\alpha_{\mathrm{sl}} / \alpha_{\mathrm{sC}}$ on $f$ for the chain of $n=950$ taking $\sigma=2.0 \times 10^{-4}$

\begin{tabular}{cccc}
\hline$f$ & $f_{\mathrm{H}}$ & $\left(\left\langle s^{2}\right\rangle_{\mathrm{l}} /\left\langle s^{2}\right\rangle_{\mathrm{C}}\right)^{1 / 2}$ & $\alpha_{\mathrm{sl}} / \alpha_{\mathrm{sC}}\left(=\alpha_{\mathrm{h}}\right)$ \\
\hline 0.050 & 0.048 & 1.0084 & 1.0 \\
0.150 & 0.142 & 1.0250 & 1.0 \\
0.250 & 0.234 & 1.0415 & 1.0 \\
0.350 & 0.325 & 1.0582 & 1.0 \\
0.450 & 0.417 & 1.0752 & 1.0 \\
0.525 & 0.486 & 1.0883 & 1.0 \\
0.600 & 0.556 & 1.1017 & 1.0 \\
0.675 & 0.628 & 1.1157 & 1.0 \\
0.750 & 0.702 & 1.1303 & 1.0 \\
0.825 & 0.778 & 1.1457 & 1.0 \\
0.875 & 0.832 & 1.1566 & 1.0 \\
0.900 & 0.860 & 1.2001 & 1.0325 \\
0.925 & 0.889 & 1.2853 & 1.1001 \\
0.950 & 0.919 & 1.4052 & 1.1963 \\
0.975 & 0.952 & 1.6094 & 1.3620 \\
\hline
\end{tabular}

expansion factor $\alpha_{\mathrm{sI}}$ in the transition region. The reason for this trend is explained in the following way: as previously mentioned, the randomly coiled sections in a fairly long chain act as flexible joints. Thus, over the range of $f_{\mathrm{H}}$ from zero to about 0.85 , the intermediate 
chain acts as if it were a random-coil of $\alpha_{\mathrm{s}}=$ $\alpha_{\mathrm{SC}}$. But in the range of $0.85<f_{\mathrm{H}} \leq 1$, the chain acts as an expanded random-coil because of additional expansion $\alpha_{\mathrm{h}}(>1)$ to $\alpha_{\mathrm{sC}}$ through the action of the helical sequences of $n_{\mathrm{h}}>160$ formed in the intermediate chain. The slowly increasing nature of $\left\langle s^{2}\right\rangle_{\mathrm{I}} /\left\langle s^{2}\right\rangle_{\mathrm{C}}$ over the range between $f_{\mathrm{H}}=0$ and $f_{\mathrm{H}} \simeq 0.85$, where $\alpha_{\mathrm{sI}}$ is constant (see the last column of Table I), is due to the slowly increasing nature of $\left\langle s^{2}\right\rangle_{\text {ol }}$ (see eq 12).

For an infinitely long chain $(n=\infty), f_{\mathrm{H}}$ and $n_{\mathrm{h}}$ are reduced to

$$
f_{\mathrm{H}}=f
$$

and

$$
n_{\mathrm{h}}=\frac{f_{\mathrm{H}}^{1 / 2}}{\sigma^{1 / 2}\left(1-f_{\mathrm{H}}\right)^{1 / 2}}
$$

The former is obtained from eq 16 whereas the latter from eq 14 and 15 . On using eq $21, n_{\mathrm{h}}$ is easily obtained at a given $f_{\mathrm{H}}$ ( $=f$ in this case), which enables one to calculate $\alpha_{\mathrm{h}}{ }^{2}$ and $\left\langle s^{2}\right\rangle_{\mathrm{I}} /$ $\left\langle s^{2}\right\rangle_{\mathrm{C}}$ from eq 20 and 19, respectively. For the case of $n=600$, the method used for making Table I is repeated to calculate $\left\langle s^{2}\right\rangle_{\mathrm{I}} /\left\langle s^{2}\right\rangle_{\mathrm{C}}$. The results for $n=\infty$ and 600 are shown in Figure 3 from which the effect of $n$ on the curve of $\left(\left\langle s^{2}\right\rangle_{\mathrm{I}} /\left\langle s^{2}\right\rangle_{\mathrm{C}}\right)^{1 / 2}$ vs. $f_{\mathrm{H}}$ is seem to be small.

Norisuye et al. ${ }^{24}$ experimentally studied the relation of $\left\langle s^{2}\right\rangle_{1}^{1 / 2} v s$. $f_{\mathrm{H}}$, where the former was obtained by light-scattering and the latter by optical rotation measurements for PBLG samples in dichloroacetic acid (DCA) containing $8.3 \mathrm{wt} \%$ cyclohexanol (CHL) at various stages of the thermal helix-coil transition. Their experimental $\left\langle s^{2}\right\rangle_{1}^{1 / 2}$ values for $\bar{N}_{w}=2180$ are plotted in Figure 4 against $f_{\mathrm{H}}$. Our results for $\left\langle s^{2}\right\rangle_{\mathrm{I}}^{1 / 2} v s . f_{\mathrm{H}}$ obtained by similar calculations used for making Table I are shown by the full curve in Figure 4 where the Norisuye et al.' $\mathrm{s}^{24}$ parameter values $\left(\left\langle s^{2}\right\rangle_{\mathrm{C}}^{1 / 2}=326 \AA\right.$ and $\sigma^{1 / 2}=$ $\left.0.85 \times 10^{-2}\right)$ were used. Figure 4 shows good agreement between the theory and experiment.

Our theoretical study on the intrinsic vis-

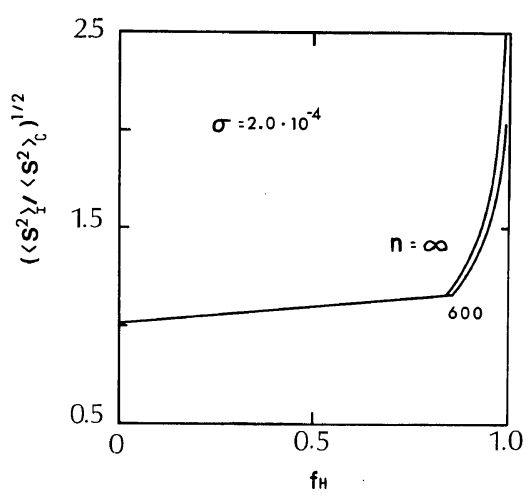

Figure 3. Dependence of $\left(\left\langle s^{2}\right\rangle_{\mathrm{I}} /\left\langle s^{2}\right\rangle_{\mathrm{C}}\right)^{1 / 2}$ on the helical content $f_{\mathrm{H}}$ for $n=\infty$ and 600 with $\sigma=2.0 \times 10^{-4}$.

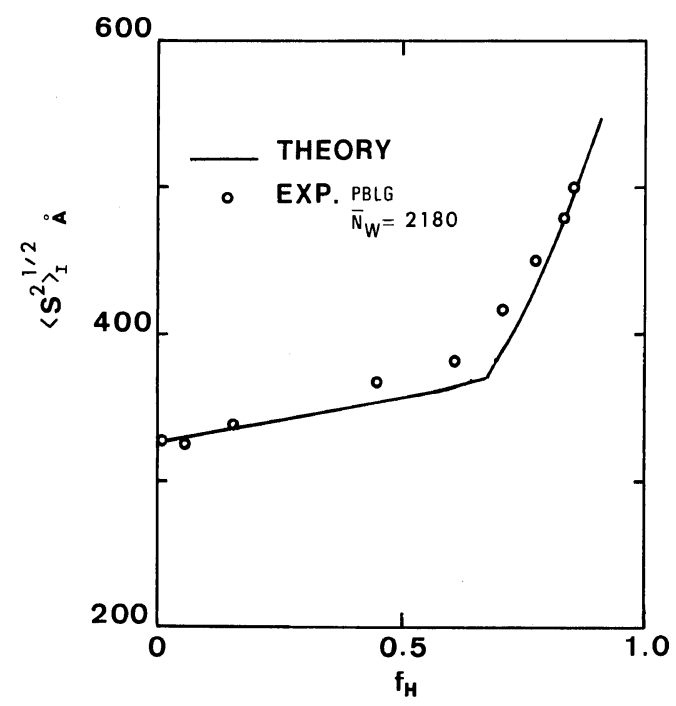

Figure 4. Variation in $\left\langle s^{2}\right\rangle_{\mathrm{I}}^{1 / 2}$ with the helical content $f_{\mathrm{H}}$. The experimental points ${ }^{24}$ are for PBLG $\left(\bar{N}_{w}=2180\right)$ in a DCA-CHL mixture $(8.3 \mathrm{wt} \% \mathrm{CHL})$.

cosity $[\eta]$ of polypeptides will be reported in Paper II.

Acknowledgement. We acknowledge with appreciation the Korea Research Center for Theoretical Physics and Chemistry for partial support of this work.

\section{REFERENCES}

1. P. Doty, A. M. Holtzer, J. H. Bradbury, and E. R. Blout, J. Am. Chem. Soc., 76, 4493 (1954); P. Doty 
and J. T. Yang, ibid., 78, 498 (1956).

2. A. Teramoto and H. Fujita, Adv. Polym. Sci., 18, 65 (1975), refer to the references cited therein.

3. J. A. Schellman, J. Phys. Chem., 62, 1485 (1958).

4. J. H. Gibbs and E. A. DiMarzio, J. Chem. Phys., 30, 271 (1959).

5. T. L. Hill, J. Chem. Phys., 30, 383 (1959).

6. B. H. Zimm and J. K. Bragg, J. Chem. Phys., 31, 526 (1959).

7. L. Peller, J. Phys. Chem., 63, 1194 (1959).

8. S. Lifson and A. Roig, J. Chem. Phys., 34, 1963 (1961).

9. K. Nagai, J. Chem. Phys., 34, 887 (1961).

10. K. Nagai, J. Phys. Soc. Jpn., 15, 407 (1960).

11. W. G. Miller and P. J. Flory, J. Mol. Biol., 15, 298 (1966).

12. A. Teramoto and H. Fujita, Adv. Polym. Sci., 18, 102 (1975).

13. D. E. Neves and R. A. Scott III, Macromolecules, 9, 554 (1976).

14. D. A. Brant and P. J. Flory, J. Am. Chem. Soc., 87, 2788 (1965).

15. D. A. Brant and P. J. Flory, J. Am. Chem. Soc., 87, 2791 (1965).
16. P. J. Flory, "Statistical Mechnics of Chain Molecules," Wiley-Interscience, New York, N.Y., 1969, (a) pp 24-26; (b) p 277; (c) pp 16-17.

17. L. Pauling, R. B. Corey, and H. R. Branson, Proc. Natl. Acad. Sci. U.S.A., 37, 205 (1951).

18. O. B. Ptitsyn and I. A. Sharanov, Zh. Tekhn. Fiz., 27, 2744 (1957).

19. C. A. J. Hoeve, J. Chem. Phys., 32, 888 (1960); see also P. J. Flory, "Statiscal Mechanics of Chain Molecules," Wiley-Intersceince, New York, N.Y., 1969, p 200.

20. P. J. Flory, "Statiscal Mechanics of Chain Molecules," Wiley-Interscience, New York, N.Y., 1969, (a) p 32; (b) p 282; (c) pp 11-12.

21. H. Yamakawa, "Modern Theory of Polymer Solutions," Harper \& Row, New York, N.Y., 1971, pp 24-26.

22. A. Teramoto, T. Norisuye, and H. Fujita, Polym. J., 1, 55 (1970).

23. K. Okita, A. Teramoto, and H. Fujita, Biopolymers, 9, 717 (1970).

24. T. Norisuye, A. Teramoto, and H. Fujita, Polym. J., 4, 323 (1973). 